\title{
Impairment of Energy Metabolism in Intact Residual Myocardium of Rat Hearts with Chronic Myocardial Infarction
}

\author{
Stefan Neubauer, Michael Horn, Anne Naumann, Rong Tian, Kai Hu, Martin Laser, Jan Friedrich, ${ }^{\ddagger}$ Peter Gaudron, \\ Klaus Schnackerz, ${ }^{*}$ Joanne S. Ingwall, ${ }^{\ddagger}$ and Georg Ertl \\ Medizinische Universitätsklinik, 97080 Würzburg, Germany; *the Theodor-Boveri-Institut für Biowissenschaften, Physiologische Chemie \\ I, Würzburg University, 97074 Würzburg, Germany; and ${ }^{\ddagger}$ the NMR Laboratory for Physiological Chemistry, Harvard Medical School, \\ Boston, Massachusetts 02115
}

\begin{abstract}
The purpose of this study was to test the hypothesis that energy metabolism is impaired in residual intact myocardium of chronically infarcted rat heart, contributing to contractile dysfunction. Myocardial infarction (MI) was induced in rats by coronary artery ligation. Hearts were isolated 8 wk later and buffer-perfused isovolumically. MI hearts showed reduced left ventricular developed pressure, but oxygen consumption was unchanged. High-energy phosphate contents were measured chemically and by ${ }^{31} \mathrm{P}-\mathrm{NMR}$ spectroscopy. In residual intact left ventricular tissue, ATP was unchanged after MI, while creatine phosphate was reduced by $31 \%$. Total creatine kinase (CK) activity was reduced by $17 \%$, the fetal $\mathrm{CK}$ isoenzymes $\mathrm{BB}$ and $\mathrm{MB}$ increased, while the "adult" mitochondrial CK isoenzyme activity decreased by $44 \%$. Total creatine content decreased by $35 \%$. Phosphoryl exchange between ATP and creatine phosphate, measured by ${ }^{31} \mathrm{P}-\mathrm{NMR}$ magnetization transfer, fell by $50 \%$ in MI hearts. Thus, energy reserve is substantially impaired in residual intact myocardium of chronically infarcted rats. Because phosphoryl exchange was still five times higher than ATP synthesis rates calculated from oxygen consumption, phosphoryl transfer via CK may not limit baseline contractile performance 2 mo after MI. In contrast, when MI hearts were subjected to acute stress (hypoxia), mechanical recovery during reoxygenation was impaired, suggesting that reduced energy reserve contributes to increased susceptibility of MI hearts to acute metabolic stress. (J. Clin. Invest. 1995. 95:1092-1100.) Key words: remodeling $\cdot{ }^{31} \mathbf{P}$-NMR - ATP $~ \cdot$ creatine phosphate $\cdot$ creatine kinase reaction velocity
\end{abstract}

Address correspondence to Stefan Neubauer, MD, Medizinische Universitätsklinik, Josef-Schneider-Strasse 2, 97080 Würzburg, Germany. Phone: 931-201-3455; FAX: 931-201-2664.

Received for publication 21 June 1994 and in revised form 26 October 1994.

1. Abbreviations used in this paper: $\mathrm{CK}$, creatine kinase; $\mathrm{CK}_{\text {mito }}$, mitochondrial isoenzyme of $\mathrm{CK}$; $\mathrm{CP}$, creatine phosphate; $\mathrm{EDP}$, end-diastolic pressure; GAP-dH, glyceraldehyde-3-phosphate dehydrogenase; $h / l$, high/low; HPLC, high-pressure liquid chromatography; LAD, left anterior descending coronary artery; LDH, lactate dehydrogenase; LVDP, left ventricular developed pressure; MI, myocardial infarction; NMR, nuclear magnetic resonance; $\mathrm{PFK}$, phosphofructokinase; $\mathrm{pHi}$, intracellular $\mathrm{pH} ; \mathrm{Pi}$, inorganic phosphate.

J. Clin. Invest.

(c) The American Society for Clinical Investigation, Inc.

0021-9738/95/03/1092/09 \$2.00

Volume 95, March 1995, 1092-1100

\section{Introduction}

The chronic hemodynamic sequelae that occur after myocardial infarction (MI) ${ }^{1}$ include left ventricular dilatation and hypertrophy of residual, intact myocardium $(1,2)$, processes described as "remodeling." Although chronic hemodynamic alterations after MI have extensively been studied both clinically and experimentally, biochemical and molecular consequences of left ventricular remodeling are only partially understood. Various compensatory and, possibly, maladaptive changes have been described at the protein or gene-expression level (e.g., references 3 and 4), but none has convincingly been linked to the development of contractile failure in post-MI heart. One attractive mechanism that may directly contribute to contractile failure after $\mathrm{MI}$ is a reduction of energy reserve (for review see reference 5). Altered energy metabolism has been observed for many experimental models of cardiac hypertrophy and failure, such as hyperthyroidism (6), aortic stenosis (7), dilated cardiomyopathy in the Syrian hamster (8), uninephrectomy plus steroid treatment (9), or the spontaneously hypertensive rat (10). The purpose of the present work was, therefore, to define performance, oxygen consumption, and parameters of energy reserve, i.e., tissue contents of ATP and creatine phosphate (CP), creatine kinase $(\mathrm{CK})$ activity and isoenzyme distribution, and phosphoryl transfer rates via CK (using ${ }^{31} \mathrm{P}$-magnetization transfer), in normal rat heart and in residual intact myocardium after MI. Using these measurements, we directly tested whether changes in energy metabolism can contribute to contractile dysfunction in post-MI heart.

\section{Methods}

Animals and experimental MI. Infarcts or sham operations were carried out in 12-wk-old Wistar rats, kept in a 12-h light-dark cycle. Left anterior descending coronary artery (LAD) ligation was performed by a previously described technique $(1,11)$. Briefly, a left thoracotomy was performed under ether anesthesia and positive pressure ventilation. The heart was rapidly exteriorized by applying gentle pressure on both sides of the thorax. The LAD was ligated between the pulmonary outflow tract and the left atrium. The heart was then replaced into the thorax, lungs were inflated by increasing positive end-expiratory pressure, and the wound was closed immediately. Sham operation was performed using an identical procedure except that the suture was passed under the coronary artery without ligation. Mortality rate of infarcted rats for the first $24 \mathrm{~h}$ after the operation was $40-50 \%$. Surviving rats were kept on commercial rat chow and water ad libitum. All procedures conformed to the guiding principles of the American Physiological Society.

Isolated rat heart preparation. $8 \mathrm{wk}$ after LAD ligation or sham operation, rats were anesthetized by injecting $20 \mathrm{mg}$ pentobarbital sodium intraperitoneally. After thoracotomy, the heart was rapidly excised and immersed in ice-cold buffer. The aorta was dissected free and mounted onto a cannula attached to a perfusion apparatus, as described previously (12). Retrograde perfusion of the heart was started in the 
Langendorff mode at a constant temperature of $37^{\circ} \mathrm{C}$ and a constant coronary perfusion pressure of $100 \mathrm{mmHg}$. A small vent made out of polyethylene tubing was pierced through the apex of the left ventricle for drainage of flow from Thebesian veins. For perfusion, phosphatefree Krebs-Henseleit buffer was used containing (mM): $\mathrm{NaCl} 118, \mathrm{KCl}$ 4.7, $\mathrm{CaCl}_{2} 1.75, \mathrm{MgSO}_{4} 1.2$, ethylenediaminetetraacetate tetrasodium $0.5, \mathrm{NaHCO}_{3} 25.0$, and glucose 11.0. Equilibrating the buffer with $95 \%$ $\mathrm{O}_{2}, 5 \% \mathrm{CO}_{2}$ yielded a $\mathrm{pH}$ of 7.4. Coronary flow was measured by an ultrasonic flow probe (Transonic Systems Inc., Ithaca, NY) built into the perfusate inflow tubing. As previously shown, the perfusion system allowed maintenance of hearts in a steady state for at least $90 \mathrm{~min}$ with changes of $<5 \%$ for all mechanical and metabolic parameters (12). For measurement of cardiac performance, a water-filled Latex balloon was inserted into the left ventricle through an incision in the left atrial appendage, via the mitral valve, and secured by a ligature. The balloon was connected to a Statham P23Db pressure transducer (Gould Instruments, Glen Burnie, MD) with a small-bore polyethylene tubing for continuous measurement of left ventricular pressure and heart rate on a 4-channel recorder (Graphtec Corp., Tokyo, Japan). Performance was estimated as the product of heart rate and left ventricular developed pressure ( $\mathrm{mmHg}$ per minute).

${ }^{31} \mathrm{P}$ nuclear magnetic resonance (NMR) spectroscopy. The perfused hearts were placed into a $20-\mathrm{mm}$ NMR sample tube and inserted into a probe seated in the bore of a superconducting super-wide-bore ( 150 $\mathrm{mm}$ ) 7.05-T magnet (Bruker, Rheinstetten, Germany). Hearts were bathed in their own perfusate which was pumped from the NMR tube at a level immediately above the heart. An Aspect 3000 computer (Bruker) was used in the pulsed Fourier transform mode to generate ${ }^{31}$ P-NMR spectra at $121.50 \mathrm{MHz}$. A 14-channel Shim Unit served to homogenize the magnetic field. Single ("one pulse") spectra were accumulated over 5-min periods, averaging data from 152 free induction decays obtained using a pulse time of $37.6 \mu \mathrm{s}$, a pulse angle of $45^{\circ}$, and an interpulse delay of $1.93 \mathrm{~s}$. The resonance areas corresponding to ATP, CP, inorganic phosphate ( $\mathrm{Pi}$ ), monophosphate esters (MPE), and NAD, which are proportional to the number of phosphorus atoms of the respective compound, were measured using the Aspect Integration Program. Relative saturation factors for each resonance were determined by comparing spectra to fully relaxed spectra obtained using a pulse angle of $45^{\circ}$ and an interpulse delay of $15 \mathrm{~s}$; correction factors were 1.12 for $\mathrm{CP}$ and 1.08 for $\mathrm{Pi}$; spectra were corrected accordingly. In each heart, the area of the $[\gamma-\mathrm{P}]$ ATP resonance of the first spectrum obtained under control conditions was arbitrarily set to $100 \%$ and used as the reference value for all resonances in the sequence of ${ }^{31} \mathrm{P}-\mathrm{NMR}$ spectra obtained for the protocol. Myocardial ATP content was found to be $28.3 \pm 5.6 \mathrm{nmol} / \mathrm{mg}$ protein in sham and $28.0 \pm 3.0 \mathrm{nmol} / \mathrm{mg}$ protein in MI hearts by HPLC ( see below); the myocardial contents of the other metabolites were calculated by multiplying the ratio of resonance area of metabolite to the $[\gamma-\mathrm{P}]$ ATP area by $28.3 \mathrm{nmol} / \mathrm{mg}$ protein for sham and by $28.0 \mathrm{nmol} / \mathrm{mg}$ protein for MI hearts. Intracellular $\mathrm{pH}$ (pHi) was measured by comparing the chemical shift between $\mathrm{Pi}$ and $\mathrm{CP}$ with values obtained from a standard curve. Free cytosolic ADP concentration was calculated assuming that $C K$ is in equilibrium and using $\mathrm{K}_{\mathrm{obs}}$ from Lawson and Veech (13).

${ }^{3 l}$ P-NMR magnetization transfer measurements of CK kinetics. For magnetization transfer experiments, each broadband pulse was preceded by a low-power, narrowband pulse at the resonance frequency of [ $\gamma$ P]ATP for $0,0.3,0.6,1.2,2.4$, or $3.6 \mathrm{~s}$ as previously described (14). Separate studies showed that the narrowband pulse directly attenuated the CP magnetization by $<5 \%$ when the carrier frequency was placed $2.5 \mathrm{ppm}$ downfield from the resonance of $\mathrm{CP}$. For each of the 6 saturation transfer spectra, 64 scans were accumulated by repetitively cycling through the 6 different times of presaturation. Thus, any metabolic deterioration occurring during the saturation transfer measurement was equally distributed among the spectra. A complete saturation transfer experiment was acquired in $32 \mathrm{~min}$. Stability of the preparation was assessed by comparing one-pulse spectra obtained before and after each magnetization transfer experiment. Magnetization transfer measurements of the forward CK reaction, creatine phosphate $\rightarrow[\gamma-\mathrm{P}]$ ATP, were analyzed according to the two-site chemical exchange model of
Forsen and Hoffman (15), providing estimates of the pseudo first-order rate constant $\left(k_{\text {for }}\right)$ and the intrinsic longitudinal relaxation time for $\mathrm{CP}$ $\left(T_{1}\right)$. Briefly, as the time of saturation at $[\gamma-P] A T P, t$, is increased from 0 to $3.6 \mathrm{~s}$, the integrated signal intensity of the $\mathrm{CP}$ resonance peak, $M_{t}$, decays from $M_{0}$ to $M_{\infty}$ (defined as magnetization at zero and infinite saturation times, respectively) with a time constant $\tau_{1}$ as follows: $\mathrm{M}_{\mathrm{t}}=\mathrm{M}_{\infty}+\left(\mathrm{M}_{0}-\mathrm{M}_{\infty}\right) \mathrm{e}^{-\mathrm{t} / \tau}$, where $1 / \tau_{1}=1 / \mathrm{T}_{1}+k_{\text {for }}$. Nonlinear regression of $\mathrm{CP}$ magnetization at different saturation times of $[\gamma-\mathrm{P}]-$ ATP was used to determine $\mathrm{M}_{0}, \mathrm{M}_{\infty}$, and $\tau_{1} . k_{\text {for }}$ and $\mathrm{T}_{1}$ were then calculated by solving the equations: $k_{\text {for }}=\left(\mathrm{M}_{0}-\mathrm{M}_{\infty}\right) /\left(\mathrm{M}_{0} \tau_{1}\right)$ and $\mathrm{T}_{1}$ $=\left(\mathbf{M}_{0} / \mathbf{M}_{\infty}\right) \tau_{1}$. Multiplying the rate constant by substrate concentration yielded reaction velocity (16). In addition, predicted $\mathrm{CK}$ reaction velocity was calculated for sham and MI hearts using the CK rate equation and literature values for $K_{\mathrm{m}}$ and $K_{\mathrm{i}}$ of substrates (17) as previously described.

High-pressure liquid chromatography measurements. Additional sham $(n=6)$ and MI $(n=6)$ hearts were isolated and perfused at 100 $\mathrm{mmHg}$ as described above. With a colored Latex balloon in the left ventricle, hearts were rapidly freeze-clamped using Wollenberger tongues as previously described (18). In freeze-clamped MI hearts, infarct size could not be determined, but at least $25 \%$ of the left ventricle was infarcted as estimated from our visual judgment of scar size; hearts with smaller infarctions were excluded. Infarct scar and intact left ventricular tissue, the border of which was marked by the colored balloon, were separated with a Minimot 40/E precise drill machine (Proxxon $\mathrm{GmbH}$, Niersbach, Germany) under liquid nitrogen and were analyzed for ATP content as previously described (18). Briefly, frozen tissue was powdered in a stainless steel percussion mortar cooled in liquid nitrogen. The powder was homogenized in $0.4 \mathrm{~N}$ perchloric acid at $0^{\circ} \mathrm{C}$, and aliquots of the homogenate were removed for protein determination. The homogenate was neutralized and centrifuged for $5 \mathrm{~min}$. The supernatant was used for measuring ATP by high-pressure liquid chromatography (HPLC) as previously described. Noncollagen protein was measured by the method of Lowry et al. (19). Metabolite concentrations were expressed as nanomoles per milligram of protein.

Enzyme activities, isoenzyme distribution, and total creatine content. Since biochemical measurements cannot be made in formalin-pretreated hearts (necessary for histologic determination of infarct size), additional sham $(n=10)$ and MI $(n=11)$ hearts were isolated and perfused for 10 min under control conditions; a biopsy of $\sim 100 \mathrm{mg}$ from the left ventricle was then immediately frozen; in MI hearts, the biopsy was taken from noninfarcted, residual left ventricular myocardium. In these MI hearts, infarct size could not be determined, but at least $25 \%$ of the left ventricle was infarcted (see above); hearts with smaller infarctions were excluded. From each sample, $10-30 \mathrm{mg}$ of tissue was homogenized in $0.1 \mathrm{M} \mathrm{K}_{2} \mathrm{HPO}_{4}$ buffer, pH 7.4, with $1 \mathrm{mM}$ EGTA, $1 \mathrm{mM} \beta$ mercaptoethanol, and $0.1 \%$ Triton at $4^{\circ} \mathrm{C}$ (final tissue concentration 5 $\mathrm{mg} / \mathrm{ml}$ ). Before the addition of Triton, aliquots were taken for measurement of protein and creatine content. Other aliquots were analyzed for total CK activity, measured at $30^{\circ} \mathrm{C}(20)$, and for CK isoenzyme distribution (21). Isoenzyme composition was measured using cellulose acetate strip electrophoresis with scanning fluorometry, taking care to prevent oxidation of the $\beta$-chain, to work in the linear range of detection and to inhibit any non-phosphocreatine-dependent source of fluorescence. Using this technique, the relative activities of all four isoenzymes are determined simultaneously. Since the specific activities of the isoenzymes are similar (22), relative activities also report relative concentrations. Total lactate dehydrogenase (LDH) activity was measured using the method of Bernstein and Everse (23). LDH high/low ratio (23), phosphofructokinase (PFK) (24), glyceraldehyde-3-phosphate dehydrogenase (GAP-dH) (25), citrate synthase activities (26), total protein (19), and total creatine content (27) were measured as previously described.

Oxygen consumption measurements. Because of the necessity to use short, metallic lines of tubing impermeable to oxygen for oxygen consumption measurements, these measurements cannot be made simultaneously with NMR measurements. Thus, we measured oxygen consumption in hearts used for enzyme analysis. Here, oxygen tension was measured in the perfusion medium at the level of the aortic cannula and 
in the coronary effluent in the right ventricle with a Clark-type electrode (Mikroprocessor oxygen meter OXI 537; WTW, Weilheim, Germany). Oxygen consumption was calculated according to the formula (28): (perfusate $\mathrm{pO}_{2}$ difference across the heart) $\times$ ( solubility of $\mathrm{O}_{2} / \mathrm{mmHg}$ ) $\times$ (coronary flow)/(dry weight in grams). In infarcted hearts, oxygen consumption was related to the weight of residual intact myocardium excluding the scar; it was assumed that oxygen consumption of scar tissue was negligible (29).

Experimental groups and protocols. For HPLC measurements, sham $(n=6)$ and $\mathrm{MI}(n=6)$ hearts were isolated, perfused for 10 min under control conditions, and rapidly freeze-clamped. For enzyme analysis and oxygen consumption measurements, sham $(n=10)$ and MI $(n=11)$ hearts were isolated, perfused for $10 \mathrm{~min}$ under control conditions, and oxygen consumption was measured. A biopsy of $\sim 100 \mathrm{mg}$ of intact left ventricle was then taken and immediately frozen. Another biopsy of $\sim 50 \mathrm{mg}$ of intact left ventricle was stored at $50^{\circ} \mathrm{C}$ for $3 \mathrm{~d}$ for determination of the wet weight/dry weight ratio (used for calculation of oxygen consumption ). For measurement of CK kinetics, hearts ( sham $=11 ; \mathrm{MI}=8$ ) were given a 10 -min stabilization period. A 5 -min onepulse spectrum was then recorded. Thereafter, a set of $6{ }^{31} \mathrm{P}-\mathrm{NMR}$ magnetization transfer spectra was recorded in $32 \mathrm{~min}$. After a final one-pulse spectrum, hearts were fixed in formalin for determination of infarct size $(1,11)$. Hearts were perfused with oxygenated KrebsHenseleit buffer throughout this protocol.

To test for increased susceptibility to acute metabolic stress, three additional groups of rat hearts were subjected to hypoxia and reoxygenation. In sham hearts $(n=7)$, end-diastolic pressure (EDP) was set to $10 \mathrm{mmHg}$ at the beginning of the experiment by adjusting left ventricular balloon volume. In one group of infarcted hearts (MI I group, $n=10$ ) EDP was also set to $10 \mathrm{mmHg}$ at the beginning of the protocol. In a second group of infarcted hearts (MI II group, $n=6$ ), EDP was first measured in vivo under ether anesthesia with a thin polyethylene catheter connected to a Statham P23Db pressure transducer (Gould Instruments) as previously described $(1,11)$; in these hearts, EDP was increased in vivo averaging $23 \mathrm{mmHg}$. EDP in the isolated, in vitro perfused heart was adjusted to the value measured before isolation by adjusting left ventricular balloon volume. Balloon volume was unchanged for the rest of the protocol. All hearts were given a 10-min stabilization period. The protocol consisted of $15 \mathrm{~min}$ of prehypoxia ( $\mathrm{pO}_{2}>500$ Torr), a 30-min period of perfusion with buffer rendered hypoxic by gassing with $95 \% \mathrm{~N}_{2}, 5 \% \mathrm{CO}_{2}\left(\mathrm{pO}_{2} \sim 20\right.$ Torr), and a 30 -min period of reoxygenation. ${ }^{31} \mathrm{P}-\mathrm{NMR}$ spectra were continuously recorded. Upon completion of the protocol, MI I and MI II hearts were fixed in formalin for determination of infarct size.

Determination of infarct size. Infarct size was determined by a previously described technique $(1,11)$. The left ventricle was embedded in paraffin, and $20-\mu \mathrm{m}$ sections were cut serialy from apex to base of the heart. Sections were stained with Masson trichrome and were mounted on slides, which were projected with a $12 \times$ multiplication. Lengths of scar and noninfarcted muscle for both endocardial and epicardial surfaces were determined by planimetry for each section. The ratio of the lengths of scar and surface circumferences defined the infarct size for endo- and epicardial surfaces, respectively. Final infarct size was determined as the average of endo- and epicardial surfaces and is given as a percentage. All hearts with an infarct size of $<25 \%$ were excluded from the analysis $(n=5)$.

Statistical analysis. Results of sham and MI hearts were compared using an unpaired $t$ test (30). In addition, for the hypoxia protocol, a two factor repeated measures ANOVA model with group (sham, MI I, MI II) as the "between" factor and time as the "within" factor was built. The analysis of changes during the protocol was restricted to three time points: control period (average of the three time points during control), end-of-hypoxia (last measurement taken during hypoxia), and steady state reoxygenation (average of the last four time points during reoxygenation). Using this model, we tested for a significant "time effect," a significant "group effect," and, in addition, the model allowed calculation of a "time versus group effect," which indicates whether the three groups respond differently over time (31). If significant main effects were proven by two factor repeated measures ANOVA,
Table I. Characteristics of Sham-operated and Infarcted Rats

\begin{tabular}{lcc}
\hline & Sham & MI \\
\hline Body weight $(\mathrm{g})$ & $555 \pm 8$ & $498 \pm 13^{*}$ \\
Heart weight $($ wet $)(\mathrm{g})$ & $1.87 \pm 0.05$ & $2.07 \pm 0.07^{*}$ \\
Heart weight/body weight $(\mathrm{g} / \mathrm{kg})$ & $3.41 \pm 0.08$ & $4.23 \pm 0.17^{*}$ \\
Infarct size $(\%)$ & - & $41 \pm 3$ \\
Heart rate $\left(\mathrm{min}^{-1}\right)$ & $299 \pm 6$ & $280 \pm 10$ \\
LVDP $(\mathrm{mmHg})$ & $97 \pm 5$ & $69 \pm 7^{*}$ \\
$\mathrm{CF}(\mathrm{ml} / \mathrm{min})$ & $26 \pm 2$ & $23 \pm 1$ \\
$\mathrm{MVO}(\mu \mathrm{mol} / \mathrm{min} \times \mathrm{g}$ dry wt) & $24 \pm 2$ & $25 \pm 2$ \\
\hline
\end{tabular}

$C F$, coronary flow; $M V O 2$, oxygen consumption. $* P<0.05$.

paired comparisons were made using a least square means test (31). In addition, for the comparison of functional recovery during reoxygenation between sham and MI I hearts, we tested percent recovery using an unpaired $t$ test (30); for sham and MI I groups, comparison of absolute rate-pressure products during reoxygenation cannot be made due to substantially different baseline values. Calculations were aided by the StatView SE + Graphics Professional, Graphic, Statistics Utility (BrainPower Inc., Calabasas, CA) and the SuperANOVA statistics utility (Abacus Concepts Inc., Berkeley, CA). All data are presented as mean \pm SE.

\section{Results}

Characteristics of sham and MI rats. Table I shows characteristics of sham operated and MI rats. Body weights were lower in rats with MI. Heart weights, and even more so, heart weight/ body weight ratios were increased in MI rats. This indicates hypertrophy of residual intact myocardium, since in MI hearts $41 \pm 3 \%$ of the left ventricle was infarcted. Infarct sizes of MI hearts from the various protocols (see Methods) were similar (hearts from MI I group $40 \pm 2 \%$, hearts from MI II group $42 \pm 4 \%$, hearts for CK kinetics $40 \pm 3 \%$ ).

Performance of sham and infarcted hearts. Table I also shows performance, coronary flow, and oxygen consumption data from sham and MI hearts studied under control conditions at an EDP of $10 \mathrm{mmHg}$. Heart rate was similar between groups. Left ventricular developed pressure (LVDP) was reduced in MI hearts $(69 \pm 7)$ compared with sham hearts $(97 \pm 5 \mathrm{mmHg}$; $P<0.05$ ), demonstrating left ventricular dysfunction. Global coronary flow (as well as coronary flow per gram of heart weight corrected for scar weight [14\%]; data not shown ) was similar for sham and MI hearts. Oxygen consumption was similar between sham and infarcted hearts.

High-energy phosphate contents and pHi. Data from Table II demonstrate, that, based on HPLC measurements, ATP content remained unchanged in residual intact myocardium of chronically infarcted hearts $(28.3 \pm 5.6 \mathrm{vs} 28.0 \pm 3.0 \mathrm{nmol} / \mathrm{mg}$ protein). In contrast, ATP content of infarct scar tissue was found to be only $0.2 \pm 0.1 \mathrm{nmol} / \mathrm{mg}$ protein, i.e., infarct scar contained almost no ATP. Figs. 1 and 2 show typical ${ }^{31}$ P-NMR spectra of a sham and an infarcted heart. In the MI heart, the $\mathrm{CP}$ resonance area is markedly reduced, while other ${ }^{31} \mathrm{P}$ resonances are similar. On average, $\mathrm{CP}$ content of chronically infarcted hearts was decreased by $31 \%$, and the CP/ATP ratio of ${ }^{31} \mathrm{P}-\mathrm{NMR}$ spectra was reduced from $1.50 \pm 0.05$ to $1.05 \pm 0.05$ $(P<0.05)$. In contrast, $\mathrm{Pi}$ content (Table II) and the Pi/ATP ratio of ${ }^{31} \mathrm{P}$-NMR spectra $(0.40 \pm 0.02$ vs $0.45 \pm 0.04$; Figs. 1 and 
Table II. High- and Low-Energy Phosphate Contents and pHi in Sham-operated and Infarcted Hearts

\begin{tabular}{lcc}
\hline & Sham & MI \\
\hline ATP (nmol/mg protein) & $28.3 \pm 5.6$ & $28.0 \pm 3.0$ \\
CP (nmol/mg protein) & $42.5 \pm 1.4$ & $29.4 \pm 1.4^{*}$ \\
$\mathrm{Pi}(\mathrm{nmol} / \mathrm{mg}$ protein) & $11.3 \pm 0.6$ & $12.6 \pm 1.1$ \\
$\mathrm{pHi}$ & $7.13 \pm 0.01$ & $7.15 \pm 0.01$ \\
Free cytosolic ADP $(\mu \mathrm{M})$ & 62 & 44 \\
\hline
\end{tabular}

$* P<0.05$, unpaired $t$ test.

2) were similar for sham and infarcted hearts. pHi was similar ( $\sim 7.14)$ for both groups. Calculation of free cytosolic ADP concentration (13) showed that free ADP does not increase in chronically infarcted hearts (62 vs $44 \mu \mathrm{M}$; sham vs MI).

CK System, citrate synthase, and glycolytic enzymes. In residual intact left ventricular tissue of MI hearts, total CK activity decreased by $17 \%$ (Table III). The CK isoenzyme distribution of noninfarcted left ventricular tissue also changed substantially after MI toward a fetal enzyme profile. The BB isoenzyme increased eightfold, while MB was increased by $62 \%$. The percentage of $\mathrm{CK}$ activity found as the MM isoenzyme was unchanged; therefore, the activity of the MM-CK isoenzyme decreased in proportion to total CK activity. Chronically infarcted hearts showed a substantial reduction of the mitochondrial CK isoenzyme $\left(\mathrm{CK}_{\text {mito }}\right)$ by $32 \%$. Since $\mathrm{CK}_{\text {mito }}$ activity is the product of percent distribution of $\mathrm{CK}_{\text {mito }}$ and of total $\mathrm{CK}$ activity, $\mathrm{CK}_{\text {mito }}$ activity was decreased by $44 \%$. The decrease of $\mathrm{CK}_{\text {mito }}$ represents a specific reduction of this enzyme and is not due to a
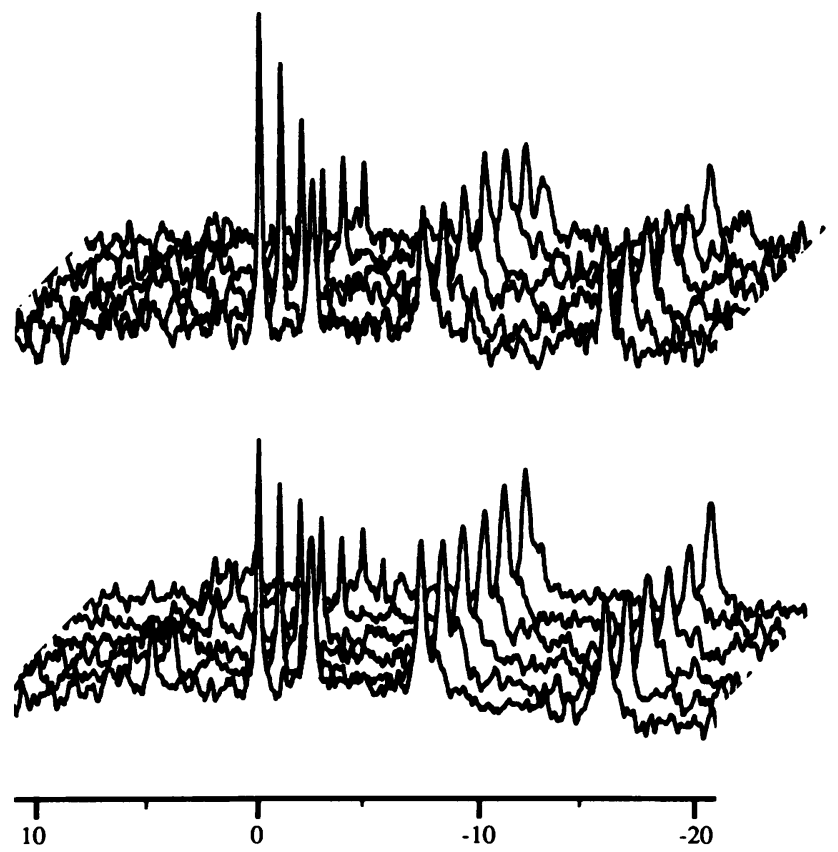

Figure 1. Magnetization transfer spectra. Stacked plots of ${ }^{31} \mathrm{P}-\mathrm{NMR}$ spectra from magnetization transfer experiments in a sham (top) and an MI heart (bottom). In each set of six magnetization transfer spectra, the duration of presaturation of the $[\gamma-P]$ ATP resonance, from front to back, is $0,0.3,0.6,1.2,2.4$, and $3.6 \mathrm{~s}$. For the sake of clarity, assignment of peaks is given in Fig. 2.

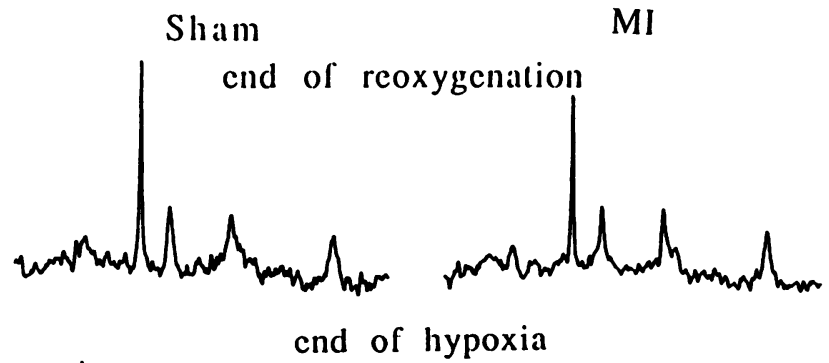

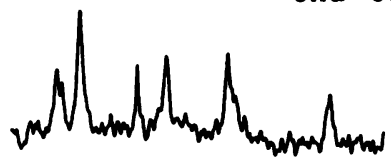

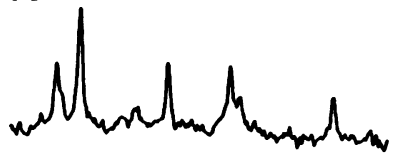

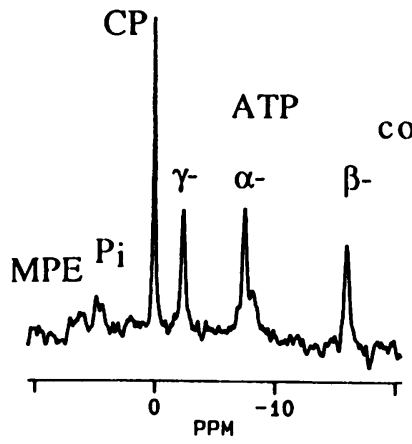

control

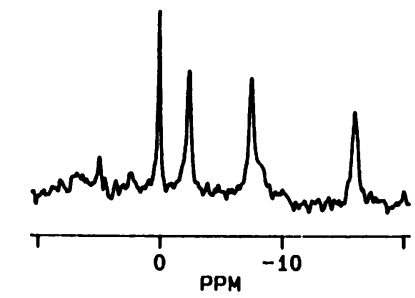

Figure 2. ${ }^{31} \mathrm{P}-\mathrm{NMR}$ spectra. Representative ${ }^{31} \mathrm{P}-\mathrm{NMR}$ spectra of a shamoperated and an infarcted heart from the MI II group (in vivo EDP) during control, at the end of hypoxia, and at the end of reoxygenation. The spectra demonstrate that the CP resonance is reduced in the infarcted heart compared with the sham heart at all stages of the protocol.

nonspecific global decrease of mitochondrial proteins, since citrate synthase, a marker enzyme for mitochondrial mass, was unchanged. Similar to the reduction of $\mathrm{CP}$, total creatine content decreased substantially by $35 \%$.

PFK and GAP-dH activities (Table III) did not change significantly after MI. LDH activity showed a significant increase by $17 \%$. The LDH isoenzyme distribution was evaluated by determining the $\mathrm{LDH}$ high/low ratio ( $\mathrm{h} / \mathrm{l}$; ratio of $\mathrm{LDH}$ activities in the presence of high and low pyruvate concentrations); an increase of the $h / l$ ratio indicates an increase of anaerobictype isoenzymes. The $\mathrm{LDH} h / 1$ ratio showed a small but sig-

Table III. CK System, Citrate Synthase, and Glycolytic Enzyme Activities in Sham-operated and Infarcted Hearts

\begin{tabular}{lcc}
\hline & Sham & MI \\
\hline Total CK (IU/mg protein) & $6.5 \pm 0.2$ & $5.4 \pm 0.3^{*}$ \\
$\% \mathrm{BB}$ & $1.1 \pm 0.3$ & $8.5 \pm 1.1^{*}$ \\
$\% \mathrm{MB}$ & $10.4 \pm 0.8$ & $16.8 \pm 0.8^{*}$ \\
$\% \mathrm{MM}$ & $56.5 \pm 1.6$ & $52.6 \pm 1.4$ \\
$\% \mathrm{mito}$ & $32.5 \pm 1.4$ & $22.1 \pm 2.1^{*}$ \\
Total creatine (nmol/mg protein) & $81.3 \pm 3.5$ & $52.7 \pm 4.1^{*}$ \\
Citrate synthase (IU/mg protein) & $0.90 \pm 0.04$ & $0.82 \pm 0.04$ \\
PFK (IU/mg protein) & $0.13 \pm 0.01$ & $0.11 \pm 0.01$ \\
GAP-dH (IU/mg protein) & $0.72 \pm 0.05$ & $0.76 \pm 0.06$ \\
LDH (IU/mg protein) & $2.9 \pm 0.1$ & $3.4 \pm 0.2^{*}$ \\
LDH h/l & $0.095 \pm 0.006$ & $0.122 \pm 0.004^{*}$ \\
\end{tabular}

*P 0.05; unpaired $t$ test. 
Table IV. CK Kinetics in Sham-operated and Infarcted Hearts

\begin{tabular}{lcc}
\hline & Sham & MI \\
\hline $\mathrm{T} 1_{\mathrm{CP}}(\mathrm{s})$ & $3.45 \pm 0.14$ & $3.41 \pm 0.36$ \\
$k\left(\mathrm{~s}^{-1}\right)$ & $0.91 \pm 0.04$ & $0.77 \pm 0.07 *$ \\
Measured CK reaction velocity $(\mathrm{mM} / \mathrm{s})$ & $10.8 \pm 0.5$ & $5.4 \pm 0.5^{*}$ \\
Predicted CK reaction velocity $(\mathrm{mM} / \mathrm{s})$ & 12.2 & 6.7
\end{tabular}

$T 1_{C P}$, longitudinal relaxation time of $\mathrm{CP} ; k$, unidirectional rate constant of the CK reaction. $* P<0.05$; unpaired $t$ test.

nificant increase in residual intact left ventricular tissue. Taken together, these results show that large changes occur in the CK system, but not in selected glycolytic or citric acid cycle enzymes.

CK kinetics in chronically infarcted heart. Fig. 1 shows sets of ${ }^{31} \mathrm{P}-\mathrm{NMR}$ magnetization transfer spectra of a sham (top) and an MI heart (bottom). The spectra demonstrate that the extent of magnetization transfer from the CP to the $[\gamma-\mathrm{P}]$ ATP resonance is reduced in the MI heart. On average (Table IV), the longitudinal relaxation time $\left(T_{1}\right)$ of $C P$ was unchanged in chronically infarcted heart. Both the rate and extent of magnetization transfer from the CP to the $[\gamma$-P]ATP resonance were reduced in MI hearts. The unidirectional rate constant $k$ for the forward CK reaction decreased from 0.91 to $0.77\left(\mathrm{~s}^{-1}\right)$. Both measured and predicted CK reaction velocity fell substantially by $50 \%$ in residual intact myocardium of chronically infarcted hearts.

Acute metabolic stress due to hypoxic perfusion. To test whether susceptibility of chronically infarcted hearts to acute metabolic stress is altered, hearts were subjected to hypoxia and reoxygenation. There were no differences for heart rate and for coronary flow among groups at any stage of this protocol (Fig. 3, $A-B$; Table V). EDP ( $\sim 10 \mathrm{mmHg}$ in sham and MI I hearts and $23 \pm 2 \mathrm{mmHg}$ in MI II hearts; see Methods ) increased monotonically during hypoxia and recovered during reoxygenation in all groups; the absence of a significant time versus group effect (0.46; Table V) demonstrates that changes of EDP during the protocol were similar for all groups. Fig. $3 D$ shows that when EDP was set to in vivo levels ( $23 \pm 2 \mathrm{mmHg}$; MI II), the rate-pressure product could be increased to match performance of sham operated hearts due to an increase of LVDP (105 \pm 3 $\mathrm{mmHg}$ ). The rate-pressure product declined during hypoxia and recovered with reoxygenation in all groups; a significant time versus group effect $(0.0001)$ indicated that the three groups behaved differently over time. The least square means test showed that recovery of the rate-pressure product during reoxygenation was greater in sham compared with MI II hearts $\left(21.5 \pm 1.1 \times 10^{3} \mathrm{mmHg} / \mathrm{min}[75 \%]\right.$ vs $16.0 \pm 0.8 \times 10^{3}$ $\mathrm{mmHg} / \mathrm{min}$ [56\%], respectively; $P=0.029$ ). In contrast, recovery of MI I hearts (compared with sham on a percent basis due to substantially different baseline rate-pressure products) was not significantly different from sham hearts $(75 \pm 2$ vs $65 \pm 5 \%$, respectively; $P=0.51$ ).

Fig. 2 shows typical ${ }^{31} \mathrm{P}-\mathrm{NMR}$ spectra of a sham and an MI heart during control, hypoxia, and reoxygenation. Fig. $3 E$ gives mean values for ATP during the protocol. In all groups, ATP declined monotonically throughout hypoxia by $25-40 \%$ (time effect 0.0001 ); although there was a trend for accelerated ATP depletion during hypoxia and for lower ATP levels during reoxygenation in MI hearts, the group effect $(0.23)$ and time
Table V. P Values of the Two-Factor Repeated Measures Analysis of Variance (Hypoxia Protocol)

\begin{tabular}{llll}
\hline & Group effect & Time effect & Time versus group effect \\
\hline HR & 0.33 & 0.0001 & 0.17 \\
CF & 0.11 & 0.0012 & 0.25 \\
EDP & 0.0002 & 0.0001 & 0.46 \\
RPP & 0.0001 & 0.0001 & 0.0001 \\
ATP & 0.23 & 0.0001 & 0.45 \\
Pi & 0.43 & 0.0001 & 0.81 \\
CP & 0.0001 & 0.0001 & 0.13 \\
pHi & 0.72 & 0.0001 & 0.62 \\
\hline
\end{tabular}

$H R$, heart rate; $C F$, coronary flow; $R P P$, rate-pressure product.

versus group effect $(0.45)$ showed that the three groups did not respond differently during the protocol. With reoxygenation, no substantial ATP recovery occurred, as is typical for high-flow hypoxic injury (18). CP (Fig. $3 F$ ) declined during hypoxia (time factor 0.0001 ) to $\sim 30 \%$ of prehypoxic values and recovered to control levels during reoxygenation. CP contents were lower in MI hearts at all stages of the protocol. However, the lack of a time versus group effect $(0.13)$ demonstrates that, despite different baseline levels, changes of CP evoked by hypoxia and reoxygenation were similar for all three groups. Fig. 3, $G$ and $H$, shows changes of $\mathrm{Pi}$ and pHi during the protocol; there were no differences among groups.

\section{Discussion}

\section{Chronic MI model}

In this study, changes in mechanical function and energy metabolism are examined in sham operated hearts and in hearts, in which a large percentage of the left ventricle was infarcted (mean 41\%). Heart weights were increased after MI, indicating hypertrophy of residual intact ventricular tissue, compensating for the loss of $\sim 40 \%$ of left ventricular myocardial mass due to infarction. Direct determination of myocardial hypertrophy in chronic myocardial infarction has been reported by others $(32,33)$, occurring at the macroscopic (increased weight) as well as the microscopic (increased myocyte length and cell volume) level.

\section{Performance and energy metabolism in chronically infarcted hearts}

Mechanical function and oxygen consumption. In chronically infarcted hearts, left ventricular dysfunction occurred. Infarcted isolated hearts had reduced LVDP, and EDP measured in vivo was elevated to $\sim 23 \mathrm{mmHg}$. Similar functional measurements of infarcted rat hearts are reported by Vleeming et al. (34) who found a systolic pressure of $111 \pm 3$ in sham and of $63 \pm 4 \mathrm{mmHg}$ in infarcted hearts in an isolated Langendorff preparation. Left ventricular dysfunction must be distinguished from myocardial contractile dysfunction in this model. At EDP of $10 \mathrm{mmHg}$, the left ventricle might not generate normal pressure simply due to increased left ventricular volume, while the single myocyte functions normally. However, Litwin and Morgan (35) recently suggested that contractile dysfunction on the myocyte level can occur in residual intact myocardium after MI. Oxygen consumption was similar ( 24 vs $25 \mu \mathrm{mol} / \mathrm{g}$ dry weight) for sham and infarcted hearts; however, this does not indicate that oxygen 
consumption is uncoupled to reduced performance. MI hearts have increased left ventricular volumes (11) and unchanged or slightly increased myocardial mass. The most adequate way to compare oxygen consumption and performance of normal and failing dilated hearts is probably via calculation of the oxygen consumption/pressure-volume area relationship $(36,37)$. Takaoka et al. (38) reported that, in patients with chronic MI, myocardial oxygen consumption and mechanical work (pressure-volume area) are closely correlated. Thus, left ventricular volume is a major determinant of oxygen consumption in chronically infarcted heart.

High-energy phosphate content. 8 wk after MI, ATP content of intact residual left ventricle was unchanged. However, the major high-energy phosphate reserve compound in heart, $\mathrm{CP}$, was reduced by $31 \%$. The reductions in CP content measured by NMR and in the total creatine pool measured using biopsy specimens $(35 \%)$ are similar, showing that the fraction of creatine which is phosphorylated is unchanged in these myocytes. As a consequence, neither the free ADP concentration ( 62 vs $44 \mu \mathrm{M}$ ) nor the ADP/ATP ratio is increased. These results show that myocytes in the noninfarcted region do not maintain normal levels of creatine. Reduced creatine and CP levels are characteristic of many models of chronic cardiac injury (6-8, 39-41).

Previous studies of the chronic infarct model $(42,43)$ have estimated changes of high-energy phosphates in whole-heart homogenates of intact and scar tissue and were, therefore, of limited value. A recent study by Sanbe et al. (29) examined high-energy phosphate stores in residual intact myocardium of rat hearts with infarct sizes similar to our work using a columnfreezing method. In agreement with our results, these authors found significant reductions of $\mathrm{CP}$ and creatine 1-12 wk after MI. In contrast, however, they also found significant reductions of ATP and of Pi. The reasons for this discrepancy are not entirely clear and may be related to the use of traditional biochemical techniques as opposed to the noninvasive tool of NMR in the present work. However, most previous studies of failing myocardium describe reduced $\mathrm{CP}$ stores in the presence of unchanged ATP content (for review see reference 5).

Glycolytic enzymes. We also found that PFK and GAP-dH activities were unaltered in remodeling left ventricular tissue, while LDH activity increased and the ratio of aerobic to anaerobic LDH isoenzymes decreased. This suggests a shift toward anaerobic metabolism. Similar observations of LDH system changes have been reported for rats with pressure-overload hypertrophy due to aortic banding (44) or in patients with chronic heart failure (45). However, observed glycolytic enzyme changes were small in magnitude, and LDH is not a rate-limiting enzyme for glycolysis. It is, thus, unlikely that these changes contribute substantially to the development of mechanical dysfunction in post-MI heart.

CK system. Previous studies have analyzed the CK system in normal, hypertrophied, and failing myocardium of various animal species and humans (for review see reference 5). In cardiac hypertrophy, the fetal isoenzymes BB and MB increase with little or no changes in other isoenzymes $(6,40,44,46)$. In the failing myocardium, reductions of total $\mathbf{C K}$ and $\mathrm{CK}_{\text {mito }}$ activity occur in addition $(6,47)$. Thus, it was postulated that increased B isoenzymes and reduced total creatine content are markers of hypertrophy while decreases of total $\mathrm{CK}$ and $\mathrm{CK}_{\text {mito }}$ are characteristic of pump failure (5). By these criteria, we observed changes in the CK system of residual intact left ventricle after MI characteristic of both hypertrophy and failure. Our results describing changes in B-CK isoenzymes during longterm remodeling supplement changes observed by Sharkey et al. (48) in coronary artery disease $3 \mathrm{wk}$ after MI in a dog model of chronic coronary occlusion. Here, a significant increase of B isoenzymes was detected in myocardium supplied by the occluded artery.

The observed changes in the CK system substantially affected the velocity of the CK reaction, which was decreased by $50 \%$. Thus, in this model of heart failure, although ATP content was unchanged, phosphoryl turnover via CK was severely reduced. The $\sim 50 \%$ decrease of $\mathrm{CK}$ reaction velocity in postMI hearts measured by NMR could be predicted by calculating the reaction velocity using the $\mathrm{CK}$ rate equation. This suggests that, in normal as well as in infarcted rat heart, the CK reaction velocity is regulated by changes in cytosolic substrate concentrations; since the cytosolic concentrations of ADP and free creatine are near their $K_{\mathrm{m}}$ values for the reaction, both ADP and creatine are limiting CK reaction velocity (14).

\section{Consequences for ventricular remodeling}

Do the observed changes in high-energy phosphate metabolism contribute to left ventricular dysfunction of chronically infarcted hearts? For baseline conditions 2 mo after MI, when mortality is low (49), it appears that high-energy phosphate metabolism is sufficiently balanced to maintain performance; altered highenergy phosphate metabolism could limit cardiac performance by reduction of the free energy change of ATP hydrolysis. However, the observations of unchanged ATP/(ADP $\cdot \mathrm{Pi})$ and $\mathrm{CP} /$ free creatine ratios suggest that the available free energy of ATP hydrolysis is unchanged at least at baseline. In infarcted hearts, phosphoryl transfer via $\mathrm{CK}$ was markedly reduced to $50 \%$ of normal values. This finding indicates reduced capacity of the CK energy shuttle, which transfers the high-energy phosphate bond from the site of ATP production (mitochondria) to the site of ATP utilization (myofibrils). By comparing the rate of ATP synthesis estimated from oxygen consumption measurements and the rate of high-energy phosphate transfer via the CK reaction, we can assess whether energy transfer becomes limiting in post-MI hearts. Assuming a P/O ratio of 3, ATP synthesis can be calculated from oxygen consumption. The ratio of CK reaction velocity to ATP synthesis rate was 11.3 in sham and 5.4 in post-MI hearts. Thus, in sham hearts, CK reaction velocity is an order of magnitude greater than maximum ATP synthesis, and in post-MI hearts it is still five times greater. This assessment suggests that at 2 mo after MI, phosphoryl transfer via CK is unlikely to limit baseline performance of residual intact myocardium. However, it is well known that remodeling processes are progressive over time (49), and it remains to be tested, therefore, whether energy metabolism becomes limiting for baseline performance at later stages after MI.

Previous studies also suggest that the CK shuttle may not be necessary to maintain a low, baseline level of performance. The CK shuttle is, however, definitely required to obtain high workloads for prolonged periods of time, i.e., to recruit the contractile reserve of the heart. In isolated rat hearts, when CK is inhibited by iodoacetamide, hearts are unable to perform at high work (50). Van Deursen et al. (51) recently reported a transgenic mouse model with a null mutation of the M-CK gene. Skeletal muscle of mutant mice showed no change in absolute muscle force, but lacked the ability to perform burst activity. Our own findings are consistent with the concept of the CK shuttle being essential for situations of acute metabolic 

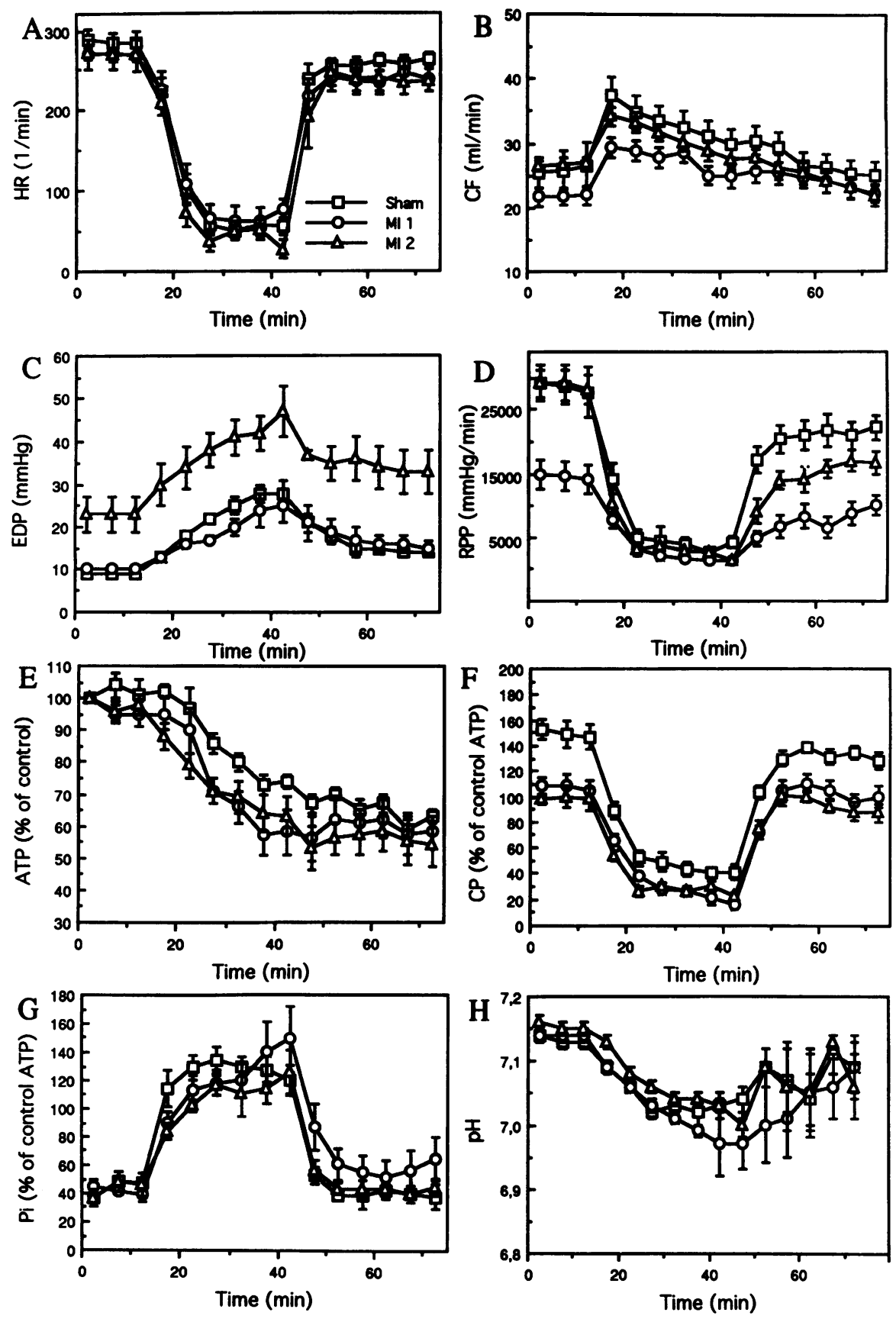

Figure 3. A-H, Cardiac performance and energy metabolism of the three groups of hearts subjected to the hypoxia/reoxygenation protocol (sham group, squares; MI I group, circles; MI II group, triangles). Changes of heart rate $(H R)$, coronary flow $(C F)$, EDP, rate-pressure product $(R P P), \mathrm{ATP}, \mathrm{CP}, \mathrm{Pi}$, and of pHi during the protocol of pre-hypoxic (0-15 $\mathrm{min})$, hypoxic (15-45 $\mathrm{min})$, and reoxygenation $(45-75 \mathrm{~min})$ periods.

stress. MI hearts showed increased susceptibility to acute stress imposed as hypoxia/reoxygenation injury, where recovery of post-hypoxic performance was impaired. In addition, when the extent of acute metabolic stress was substantially reduced by lowering pre-hypoxic workload (MI 1 hearts), infarcted hearts no longer showed impaired post-hypoxic performance. Thus, our data suggest that chronically infarcted hearts working under in vivo preload conditions lack the ability to adequately adapt to conditions of acute metabolic stress.

\section{Limitations of the model}

Using global left ventricular measures (balloon-in-LV), mechanical performance was estimated as heart rate, left ventricular developed pressure and as the rate-pressure product. Future studies combining NMR spectroscopy with NMR imaging or echocardiographic methods should allow determination of left ventricular volumes, wall thickness, wall stress, and also differences of regional function, which is anticipated to vary in left ventricle with a large a- or dyskinetic portion, depending on the distance to the scar region. Also, to exclude interactions between infarcted and noninfarcted tissue, it would be interesting to study energy metabolism under baseline and stressed conditions in a model where the effects of altered geometry on performance are of minor importance, such as the isolated papillary muscle. Saturation transfer measurements of CK reaction velocity are, however, difficult to perform on small amounts of myocardial tissue.

Global coronary flow and coronary flow per gram of viable tissue were similar for sham and infarcted hearts. It is, thus, unlikely, that observed changes of function and metabolism 
were related to changes in myocardial perfusion. However, since regional blood flow was not measured, we cannot rule out that changes of regional blood flow in infarcted hearts, e.g., altered endo/epicardial flow ratios or reduced flow in myocardium immediately adjacent to the scar, may contribute to observed changes to a certain extent.

Enzyme and HPLC analyses were performed on intact residual left ventricular tissue, but ${ }^{31} \mathrm{P}-\mathrm{NMR}$ spectra represent average metabolic estimates for the entire heart including intact and scarred myocardium. It is, however, certain, that ${ }^{31} \mathrm{P}-\mathrm{NMR}$ spectra closely approximate changes of high-energy phosphates occurring in residual intact myocardium. This is because ATP content in myocardial scar tissue assayed with HPLC was practically zero and, therefore, the contribution of scar tissue to the ${ }^{31} \mathrm{P}-\mathrm{NMR}$ signal should be negligible. The possibility remains, however, that spatial differences in high-energy phosphate contents and/or ratios exist for regions adjacent to versus remote from the scar, respectively, or for hypertrophied left versus right ventricle. Preliminary results suggest that these questions may be addressed using ${ }^{31} \mathrm{P}$-NMR spectroscopic imaging techniques $(52,53)$.

In summary, we have shown that in chronically infarcted rats 2 mo after MI, left ventricular function, CP, creatine, total $\mathrm{CK}$ and $\mathrm{CK}_{\text {mito }}$ activity are reduced, and the $\mathrm{CK}$ reaction velocity decreases by $50 \%$; oxygen consumption remains unchanged. Analysis of ATP synthesis rates calculated from oxygen consumption and from CK reaction velocity showed that phosphoryl transfer via CK may not limit baseline performance of chronically infarcted hearts. However, challenge with acute hypoxia showed that the ability of chronically infarcted hearts to respond to conditions of acute metabolic stress is limited.

\section{Acknowledgments}

The authors highly appreciate technical assistance of Ilana Reis and Anette Busch and advice on statistical analysis from John B. Newell, Cardiac Computer Center, Massachusetts General Hospital, Boston.

This work was supported by grant SFB 355/A-3 and B-1 from the Deutsche Forschungsgemeinschaft, National Institutes of Health grant HL-43170, and by a grant from the Sandoz-Stiftung, Nürnberg, Germany.

\section{References}

1. Pfeffer, M. A., J. M. Pfeffer, M. C. Fishbein, P. J. Fletcher, J. Spadaro, R. A. Kloner, and E. Braunwald. 1979. Myocardial infarct size and ventricular function in rats. Circ. Res. 44:503-512.

2. Pfeffer, J. M., M. A. Pfeffer, P. J. Fletcher, and E. Braunwald. 1991. Progressive ventricular remodeling in rat with myocardial infarction. Am. J. Physiol. 260:H1406-H1414.

3. Bilger, J., H. Reinecke, R. Studer, J. Holtz, and H. Drexler. 1993. Altered myocardial gene expression of $\mathrm{Ca}^{2+}$-ATPase and $\mathrm{Na}^{+} / \mathrm{Ca}^{2+}$-exchanger early after infarction: normalization in the chronic healing phase despite persistent up-regulation of ANF-expression. Circulation. 88:I-246a. (Abstr.)

4. Michel, J. B., J. L. Lattion, M. L. Cerol, M. Philippe, J. P. Camilleri, and P. Corvol. 1988. Hormonal and cardiac effects of converting enzyme inhibition in rat myocardial infarction. Circ. Res. 62:641-650.

5. Ingwall, J. S. 1993. Is cardiac failure a consequence of decreased energy reserve? Circulation. 87(Suppl. VII):VII-58-VII-62.

6. Ingwall, J. S., D. E. Atkinson, K. Clarke, and J. K. Fetters. 1990. Energetic correlates of cardiac failure: changes in the creatine kinase system in the failing myocardium. Eur. Heart J. II(Suppl. C):108-115.

7. Zhang, J., M. Yoshiyama, K. Hendrich, H. Liu, H. Merkle, M. Garwood, A. H. L. From, R. J. Bache, and K. Ugurbil. 1991. Myocardial bioenergetics in the hypertrophied left ventricle studied by transmural P-31 NMR spectroscopy. Proc. 1Oth Annu. Meet. SMRM. 2:911a. (Abstr.)

8. Markiewicz, W., S. S. Wu, W. W. Parmley, C. B. Higgins, R. Sievers, T. L. James, J. Wikman-Coffelt, and G. Jasmin. 1986. Evaluation of the hereditary
Syrian hamster cardiomyopathy by ${ }^{31} \mathrm{P}$ nuclear magnetic resonance spectroscopy: improvement after acute verapamil therapy. Circ. Res. 59:597-604.

9. Wexler, L. F., B. H. Lorell, S. Momomura, E. O. Weinberg, J. S. Ingwall, and C. S. Apstein. 1988. Enhanced sensitivity to hypoxia-induced diastolic dysfunction in pressure-overload left ventricular hypertrophy in the rat: role of highenergy phosphate depletion. Circ. Res. 62:766-775.

10. Shimamoto, N., N. Goto, M. Tanabe, T. Imamoto, S. Fujiwara, and M. Hirata. 1982. Myocardial energy metabolism in the hypertrophied hearts of spontaneously hypertensive rats. Basic Res. Cardiol. 77:359-371.

11. Gaudron, P., M. Blumrich, and G. Ertl. 1993. Aggravation of left ventricular dilatation and reduction of survival by a calcium channel blocker in rats with chronic myocardial infarction. Am. Heart J. 125:1226-1233.

12. Neubauer, S., G. Ertl, U. Haas, F. Pulzer, and K. Kochsiek. 1990. Effects of Endothelin-1 in the isolated perfused rat heart. J. Cardiovasc. Pharmacol. 16:1-8.

13. Lawson, J. W. R., and R. L. Veech. 1979. Effects of $\mathrm{pH}$ and free $\mathrm{Mg}^{2+}$ on the $\mathrm{K}_{\mathrm{eq}}$ of the creatine kinase reaction and other phosphate hydrolyses and phosphate transfer reactions. J. Biol. Chem. 254:6528-6537.

14. Neubauer, S., B. L. Hamman, S. B. Perry, J. A. Bittl, and J. S. Ingwall. 1988. Velocity of the creatine kinase reaction decreases in post-ischemic myocardium. A ${ }^{31}$ P-NMR magnetization transfer study of the isolated ferret heart. Circ. Res. 63:1-15.

15. Forsen, S., and R. A. Hoffman. 1963. Study of moderately rapid chemical exchange reactions by means of nuclear magnetic double resonance. J. Chem. Phys. 39:2892-2901.

16. Bittl, J. A., and J. S. Ingwall. 1985. Reaction rates of creatine kinase and ATP synthesis in the isolated rat heart. A ${ }^{31} \mathrm{P}-\mathrm{NMR}$ magnetization transfer study. J. Biol. Chem. 260:3512-3517.

17. Mc Auliffe, J. J., S. B. Perry, E. E. Brooks, and J. S. Ingwall. 1993. Kinetics of the creatine kinase reaction in neonatal rabbit heart: an empirical analysis of the rate equation. Biochemistry. 30:2585-2593.

18. Neubauer, S., and J. S. Ingwall. 1989. Verapamil attenuates ATP depletion during hypoxia by reducing pre-hypoxic cardiac performance: ${ }^{31} \mathrm{P}-\mathrm{NMR}$ studies of isolated rat heart. J. Mol. Cell. Cardiol. 21:1163-1178.

19. Lowry, O. H., N. J. Rosebrough, A. L. Farr, and R. J. Randall. 1951. Protein measurement with the Folin phenol reagent. J. Biol. Chem. 193:265-275.

20. Rosalki, S. B. 1967. An improved procedure for serum creatine phosphokinase determination. J. Lab. Clin. Med. 69:696-705.

21. Hall, N., and M. DeLuca. 1976. Electrophoretic separation and quantitation of creatine kinase isozymes. Anal. Biochem. 76:561-567.

22. Basson, C. T., A. M. Grace, and R. Roberts. 1985. Enzyme kinetics of a highly purified mitochondrial creatine kinase in comparison with cytosolic forms. Mol. Cell. Biochem. 67:151-159.

23. Bernstein, L. H., and J. Everse. 1975. Determination of the isoenzyme levels of lactate dehydrogenase. Methods Enzymol. 41:47-52.

24. Oblinger, M. M., L. G. Foe, D. Kwiatkowska, and R. G. Kemp. 1988. Phosphofructokinase in the rat nervous system: regional differences in activity and characteristics of axonial transport. J. Neurosci. Res. 21:25-34.

25. GAPdH: Methods of Enzymatic Analysis. 1974. Edited by Hans Ulrich Bergmeyer, in collaboration with Karlfried Gawehn. Second English Edition. Volume 1. 466-467.

26. Srere, P. A., H. Brazil, and L. Gowen. 1963. The citrate condensing enzyme of pigeon breast muscle and moth flight muscle. Acta Chem. Scand. 17(Suppl. 1):S129-S134.

27. Kammermeier, H. 1973. Microassay of free and total creatine from tissue extracts by combination of chromatographic and fluorometric methods. Anal. Biochem. 56:341-345.

28. Neely, J. R., H. Liebermeister, E. J. Battersby, and H. E. Morgan. 1967. Effect of pressure development on oxygen consumption by isolated rat heart. Am. J. Physiol. 212:804-815.

29. Sanbe, A., K. Tanonaka, Y. Hanaoka, T. Katoh, and S. Takeo. 1993. Regional energy metabolism of failing hearts following myocardial infarction. $J$. Mol. Cell. Cardiol. 25:995-1013.

30. Zar, J. H. 1974. Biostatistical Analysis. Prentice Hall, Englewood Cliffs, NJ. 130-181.

31. Winer, B. J. 1971. Statistical Principles in Experimental Design. Chapter 7. McGraw-Hill, New York.

32. Anversa, P., C. Beghi, Y. Kikkawa, and G. Olivetti. 1986. Myocardial infarction in rats: infarct size, myocyte hypertrophy, and capillary growth. Circ. Res. 58:26-37.

33. Zimmer, H. G., A. M. Gerdes, S. Lortet, and G. Mall. 1990. Changes in heart function and cardiac cell size with chronic myocardial infarction. J. Mol. Cell. Cardiol. 22:1231-1243.

34. Vleeming, W., H. H. van Rooij, J. Wemer, and A. J. Porsius. 1991. Cardiovascular responses to the stereoisomers of dobutamine in isolated rat hearts 48 hours after acute myocardial infarction. J. Cardiovasc. Pharmacol. 17:634640.

35. Litwin, S. E., and J. P. Morgan. 1992. Captopril enhances intracellular calcium handling and $\beta$-adrenergic responsiveness of myocardium from rats with postinfarction failure. Circ. Res. 71:797-807.

36. Wolff, M. R., P. P. de Tombe, Y. Harasawa, D. Burkhoff, S. Bier, W. C. 
Hunter, G. Gerstenblith, and D. A. Kass. 1992. Alterations in left ventricular mechanics, energetics and contractile reserve in experimental heart failure. Circ. Res. 70:516-529.

37. Nozawa, T., C. P. Cheng, T. Noda, and W. C. Little. 1994. Relation between left ventricular oxygen consumption and pressure-volume area in conscious dogs. Circulation. 89:810-817.

38. Takaoka, H., M. Takeuchi, M. Odake, Y. Hayashi, K. Hata, M. Mori, and M. Yokoyama. 1993. Comparison of hemodynamic determinants for myocardia oxygen consumption under different contractile states in human ventricle. Circula tion. 87:59-69.

39. De Tombe, P. P., M. R. Wolff, D. A. Kass, W. C. Hunter, I. Reis, and J. S. Ingwall. 1991. Creatine kinase isoenzyme expression is altered in experimental canine dilated cardiomyopathy without hypertrophy. Circulation. 84:II-280a. (Abstr.)

40. Ingwall, J. S., M. F. Kramer, M. A. Fifer, B. H. Lorell, R. Shemin, W Grossman, and P. D. Allen. 1985. The creatine kinase system in normal and diseased myocardium. N. Engl. J. Med. 313:1050-1054.

41. Nascimben, L., P. Pauletto, A. C. Pessina, I. Reis, and J. S. Ingwall. 1991 Decreased energy reserve may cause pump failure in human dilated cardiomyopathy. Circulation. 84:II-563a. (Abstr.)

42. Bester, A. J., E. Bajusz, and A. Lochner. 1972. Effect of ischemia an infarction on the metabolism and function of the isolated, perfused rat heart. Cardiovasc. Res. 6:284-294.

43. Fellenius, E., C. A. Hansen, O. Mjos, and J. R. Neely. 1985. Chronic infarction decreases maximum cardiac work and sensitivity of the heart to extracellular calcium. Am. J. Physiol. 249:H80-H87.

44. Vatner, D. E., and J. S. Ingwall. 1984. Effects of moderate pressure overload cardiac hypertrophy on the distribution of creatine kinase isozymes. Proc. Soc. Exp. Biol. Med. 175:5-9.
45. Schultheiss, H. P., G. Ullrich, M. Schindler, K. Schulze, and B. E. Strauer. 1990. The effect of ACE inhibition on myocardial energy metabolism. Eur. Hear J. 11 (Suppl. B):116-122.

46. Meerson, F. Z., and M. P. Javich. 1982. Isoenzyme pattern and activity of myocardial creatine phosphokinase under heart adaptation to prolonged overload. Basic Res. Cardiol. 77:349-358.

47. Ingwall, J. S., and E. T. Fossel. 1983. Changes in the creatine kinase system in the hypertrophied myocardium of the dog and rat. In Perspectives in Cardiovascular Research. Vol. 7. Myocardial Hypertrophy and Failure. N. R. Alpert, editor. Raven Press, New York. 601-617.

48. Sharkey, S. W., M. M. Murakami, S. A. Smith, and F. S. Apple. 1991. Canine myocardial creatine kinase isoenzymes after chronic coronary artery occlusion. Circulation. 84:333-340.

49. Pfeffer, J. M., M. A. Pfeffer, P. J. Fletcher, and E. Braunwald. 1991 Progressive ventricular remodeling in rat with myocardial infarction. Am. J. Physiol. 260:H1406-H1414.

50. Hamman, B. L., J. A. Bittl, W. E. Jacobus, and J. S. Ingwall. 1986. Inhibition of creatine kinase decreases the contractile reserve of the isolated rat heart. Proc. 5th Annu. Meet. SMRM. WIP:133.

51. van Deursen, J., A. Heerschap, F. Oerlemans, W. Ruitenbeek, P. Jap, H. ter Laak, and B. Wieringa. 1993. Skeletal muscles of mice deficient in muscle creatine kinase lack burst activity. Cell. 74:621-631.

52. von Kienlin, M., A. Metzler, F. Roder, A. Haase, M. Horn, B. Braeker, S. Neubauer, and G. Ertl. 1993. Three-dimensional ${ }^{31} \mathrm{P}$ spectroscopic imaging of regional high-energy phosphate metabolism in chronic myocardial infarction. Proc. 12th Annu. Meet. SMRM. I-357.

53. Friedrich, J., S. Ngoy, C. Apstein, and J. S. Ingwall. 1993. ${ }^{31} \mathrm{P}$ spectroscopic imaging of remodeled myocardium of the infarcted heart. Circulation. 88(Suppl.):I-379a. (Abstr.) 